\title{
$k$-Zumkeller labeling of super subdivision of some graphs
}

\author{
M. Basher ${ }^{*}$
}

\section{${ }^{*}$ Correspondence:}

m_e_basher@yahoo.com Department of Mathematics and Computer Science, Faculty of Science, Suez University, Suez, Egypt

\begin{abstract}
A simple graph $G=(V, E)$ is said to be $k$-Zumkeller graph if there is an injective function $f$ from the vertices of $G$ to the natural numbers $N$ such that when each edge $x y \in E$ is assigned the label $f(x) f(y)$, the resulting edge labels are $k$ distinct Zumkeller numbers. In this paper, we show that the super subdivision of path, cycle, comb, ladder, crown, circular ladder, planar grid and prism are $k$-Zumkeller graphs.
\end{abstract}

Keywords: Zumkeller number, k-Zumkeller labeling, Complete bipartite graph, Super subdivision

Mathematics Subject Classification: 05C, 05C78, 11 Axx

\section{Introduction}

Graph labeling was first introduced by Alex Rosa in the mid-sixties [1]. It concerns the assignment of mathematical objects, such as integers, prime numbers, modular integers, element of group, etc. The properties of the mathematical object are used through an evaluating function that assigns the values to the edges and/or vertices of graph $G=(V, E)$ based upon certain criteria. There are enormous papers have been devoted to several kinds of labeling of graphs over the past three decades, which are updated by Gallian [2]. Labeled graphs are used in numerous areas like coding theory, X-ray crystallography, the design of good radar type codes, astronomy, circuit design, communication network addressing, data base management [2]. Graphs with labeled edges are frequently used to model networks, with restrictions on the network represented as restrictions on the labels of edges. For example, when modeling transportation networks, such labels can be used to indicate various factors, from cost to level of traffic stream. In generally Ahuja, Magnati and Orlin [3] point out different applications in statistical physics, particle physics, computer science, biology, economics, operations research and sociology. For all notations and terminology in graph theory we follow Harary [4].

Graph labeling is a strong relation between number theory and graph structures. The study of Zumkeller numbers [5] is a part of number theory which is one of the important branches of mathematics. Some parts of number theory play an important role in modern coding and cryptography. Balamurugan et al. [6] introduced Zumkeller labeling using Zumkeller numbers, which is defined as an injective function $f: V \rightarrow N$ author(s) and the source, provide a link to the Creative Commons licence, and indicate if changes were made. The images or other third party material in this article are included in the article's Creative Commons licence, unless indicated otherwise in a credit line to the material. If material is not included in the article's Creative Commons licence and your intended use is not permitted by statutory regulation or exceeds the permitted use, you will need to obtain permission directly from the copyright holder. To view a copy of this licence, visit http:// creativecommons.org/licenses/by/4.0/. 
such that the induced function $f^{*}: E \rightarrow N$ defined by $f^{*}(x y)=f(x) f(y)$ is a Zumkeller number for all $x y \in E, x, y \in V$. The concept of $k$-Zumkeller labeling of graphs has been introduced and investigated in the literature $[7,8]$.

Definition 1.1 [7] A function $f$ is called $k$-Zumkeller labeling of the graph $G$ if $f: V(G) \rightarrow N$ is injective and the induced function $f^{*}: E \rightarrow N$ defined by $f^{*}(u v)=f(u) f(v)$ is a Zumkeller number for all $u v \in E(G), u, v \in V(G)$ and the resulting edge labels are $k$ distinct Zumkeller numbers.

In 2001, Sethuraman and Selvaraju [9] have introduced a graph operation called super subdivision of graph, denoted $\operatorname{SSD}(G)$ if $\operatorname{SSD}(G)$ is obtained from $G$ by replacing every edge $x y$ of $G$ by a complete bipartite graph $K_{2, t}$ by in such a way that the end vertices $x, y$ of each edge are merged with the two vertices of 2-vertices part of $K_{2, t}$ after removing the edge $x y$ from $G$ (in the complete bipartite graph $K_{2, t}$ the part consisting of two vertices is referred as 2-vertices part of $K_{2, t}$ and the part consisting of $t$ vertices is referred as $t$-vertices part of $\left.K_{2, t}\right)$. In graph theory, subdivision is a significant aspect that allows one to calculate properties of some complex graphs in terms of some easier graphs. Subdivision graphs are used to drive many mathematical and chemical properties of more complex graphs from more basic graphs and there are many results on these graphs, so it helps to study the physical, chemical properties of the object which is modeled by the graph [10].

\section{Zumkeller numbers: definition and properties}

In this section, we survey the notations of Zumkeller numbers and some properties of Zumkeller numbers. A positive integer $n$ is known as a perfect number if the sum of its proper positive factors is equal to $n$. Generalizing the notion of perfect numbers, Zumkeller presented in Encyclopedia of Integer Sequences [5] A083207 a sequence of integers that their positive factors can be separated into two disjoint subsets with equal sum.

Definition 2.1 Let $n$ be a positive number, if all the positive factors of $n$ can be partitioned into two disjoint subsets such that the sums of the two subsets are equal. Then $n$ is called a Zumkeller number.

This partition is called as a Zumkeller partition. For example, all the following numbers 6, 12, 20, 24, 28, 30 are Zumkeller numbers.

Properties of Zumkeller Numbers:

1. If the prime factorization of an even Zumkeller number $n$ is $2^{k} p_{1}^{k_{1}} p_{2}^{k_{2}} \ldots p_{m}^{k_{m}}$. Hence, at least one of $k_{i}$ must be an odd number.

2. Let $n$ be a Zumkeller number and $p$ be a prime where $(n, p)=1$, then $n p^{\ell}$ is a Zumkeller number for any positive integer $\ell$.

3. If $p_{1}^{k_{1}} p_{2}^{k_{2}} \ldots p_{m}^{k_{m}}$ is the prime factorization of the Zumkeller number $n$. Hence, for any positive integers $\ell_{1}, \ell_{2}, \ldots, \ell_{m}$. The number $p_{1}^{k_{1}+\ell_{1}\left(k_{1}+1\right)} p_{2}^{k_{2}+\ell_{2}\left(k_{2}+1\right)} \ldots p_{m}^{k_{m}+\ell_{m}\left(k_{m}+1\right)}$ is also Zumkeller number. 


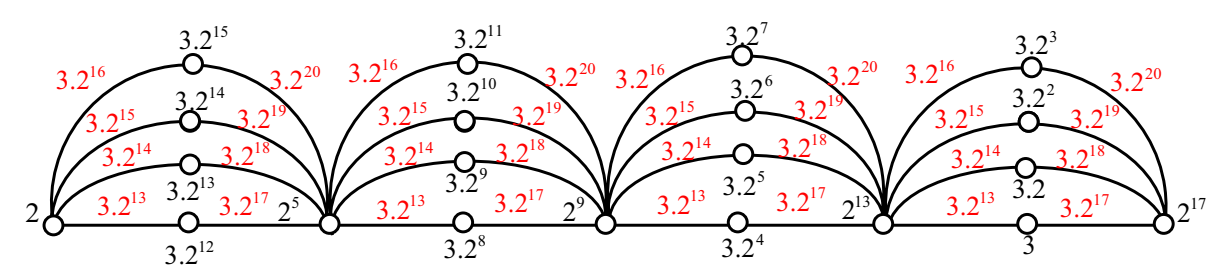

Fig. 1 8-Zumkeller labeling of $\operatorname{SSD}\left(P_{5}\right)$

4. Let $p \neq 2$ be a prime number and let $k$ be a positive integer with $p \leq 2^{k+1}-1$. Then, $2^{k} p$ is a Zumkeller number.

\section{Main results}

In this section, we prove that the super subdivision of graphs such as path $P_{n}$, cycle $C_{n}$, comb $P_{n} \odot K_{1}$, crown $C_{n} \odot K_{1}$, ladder $L_{n}$, circular ladder $C L_{n}$, planer grid $P_{m} \times P_{n}$ and prism $C_{m} \times P_{n}$ are $k$-Zumkeller graphs.

Theorem 3.1 $\operatorname{SSD}\left(P_{n}\right)$ admits a 2 -Zumkeller labeling for all $n \geq 2$.

Proof Let $P_{n}$ be a path with vertex set $V=\left\{u_{i}: 1 \leq i \leq n\right\}$ and edge set $E=\left\{e_{i}=u_{i} u_{i+1}: 1 \leq i \leq n-1\right\}$. Then, the super subdivision of the path $P_{n}, \operatorname{SSD}\left(P_{n}\right)$ is obtained by replacing each edge $e_{i}$ by a complete bipartite graph $K_{2, t}$ for some $t \in N$. Let $u_{i(i+1)}^{k}(1 \leq i \leq n-1,1 \leq k \leq t)$ be the vertices of $t$-vertex part of the complete bipartite $K_{2, t}$. From the constructions, we observe that the graph $\operatorname{SSD}\left(P_{n}\right)$ has $(n-1) t+n$ vertices and $2(n-1) t$ edges, where $t$ is the number of vertices in the $t$-vertices part of $K_{2, t}$. We define $f: V\left(\operatorname{SSD}\left(P_{n}\right)\right) \rightarrow N$ as follows:

For $1 \leq k \leq t$

$$
\begin{aligned}
f\left(u_{i}\right) & =2^{t i+(1-t)}, 1 \leq i \leq n \\
f\left(u_{i(i+1)}^{k}\right) & =p 2^{t(n-1)-t i+(k-1)}, 1 \leq i \leq n-1 .
\end{aligned}
$$

where $p<10, p \neq 2$ is a prime number. Hence the labels of the edges of $\operatorname{SSD}\left(P_{n}\right)$ are given as follows:

$$
\begin{aligned}
& f^{*}\left(u_{i} u_{i(i+1)}^{k}\right)=p 2^{t(n-2)+k}, \\
& f^{*}\left(u_{i(i+1)}^{k} u_{(i+1)}\right)=p 2^{t(n-1)+k} .
\end{aligned}
$$

From Eqs. (1), (2), it is observed that the edges of $\operatorname{SSD}\left(P_{n}\right)$ receive only $2 t$ distinct Zumkeller numbers $p 2^{t(n-2)+k}, p 2^{t(n-1)+k}$ for $1 \leq k \leq t$. Hence $\operatorname{SSD}\left(P_{n}\right)$ graph admits a $2 t$-Zumkeller labeling.

Illustration 3.1 Figure 1 illustrates the super subdivision of $P_{5}$ and its 8-Zumkeller labeling, where $t=4$ and $p=3$. 
Theorem 3.2 $\operatorname{SSD}\left(C_{n}\right)$ admits a $3 t$-Zumkeller labeling for all $n \geq 2$.

Proof Let $C_{n}$ be a cycle with vertices set $V=\left\{u_{i}: 1 \leq i \leq n\right\}$ and edge set $E=\left\{e_{i}=u_{i} u_{i+1}: 1 \leq i \leq n-1\right\} \bigcup\left\{e_{n}=u_{n} u_{1}\right\}$. Let $\operatorname{SSD}\left(C_{n}\right)$ be a super subdivision of the cycle $C_{n}$. Let $u_{i(i+1)}^{k}$ and $u_{n 1}^{k}(1 \leq i \leq n-1,1 \leq k \leq t)$ be the vertices of the $t$-vertices part of $K_{2, t}$ which are used for super subdivision of $C_{n}$. Thus $\operatorname{SSD}\left(C_{n}\right)$ has $p=n(1+t)$ vertices and $q=2 n t$ edges. We define $f: V\left(\operatorname{SSD}\left(C_{n}\right)\right) \rightarrow N$ as follows:

For $1 \leq k \leq t$.

$$
\begin{aligned}
f\left(u_{i}\right) & =2^{t i+(1-t)}, 1 \leq i \leq n \\
f\left(u_{i(i+1)}^{k}\right) & =p 2^{t n-t i+(k-1)}, 1 \leq i \leq n-1 \\
f\left(u_{n 1}^{k}\right) & =p 2^{k-1} .
\end{aligned}
$$

where $p<10, p \neq 2$ is a prime number. Then the labels of the edges of $\operatorname{SSD}\left(C_{n}\right)$ are given as follows:

For $1 \leq i \leq n-1$.

$$
\begin{aligned}
& f^{*}\left(u_{i} u_{i(i+1)}^{k}\right)=f^{*}\left(u_{n} u_{n 1}^{k}\right)=p 2^{t(n-1)+k}, \\
& f^{*}\left(u_{i(i+1)}^{k} u_{(i+1)}\right)=p 2^{t n+k}, \\
& f^{*}\left(u_{n 1}^{k} u_{1}\right)=p 2^{k} .
\end{aligned}
$$

From Eqs. (3) to (5), it is observed that the edges of $\operatorname{SSD}\left(C_{n}\right)$ receive only $3 t$ distinct Zumkeller numbers $p 2^{t(n-1)+k}, p 2^{t n+k}$ and $p 2^{k}$ for $1 \leq k \leq t$. Hence $\operatorname{SSD}\left(C_{n}\right)$ graph admits a $3 t$-Zumkeller labeling.

Illustration 3.2 Figure 2 illustrates the super subdivision of $C_{8}$ and its 9-Zumkeller labeling, where $t=3$ and $p=3$.

Theorem 3.3 $\operatorname{SSD}\left(P_{n} \odot K_{1}\right)$ admits a 3t-Zumkeller labeling for all $n \geq 3$.

Proof Let $V=\left\{u_{i}, v_{i}: 1 \leq i \leq n\right\}$ be the vertex set and $E=\left\{e_{i}=u_{i} u_{i+1}, \hat{e}_{i}=u_{i} v_{i}: 1 \leq i \leq n-1\right\} \bigcup\left\{\hat{e}_{n}=u_{n} v_{n}\right\}$ be the edge set of the comb graph. Suppose that $u_{i(i+1)}^{k}, w_{i}^{k}(1 \leq i \leq n-1,1 \leq k \leq t)$ and $w_{n}^{k}$ are the vertices of the $t$-vertices part of $K_{2, t}$ which are used for super subdivision of $P_{n} \odot K_{1}$. Hence, we observe 


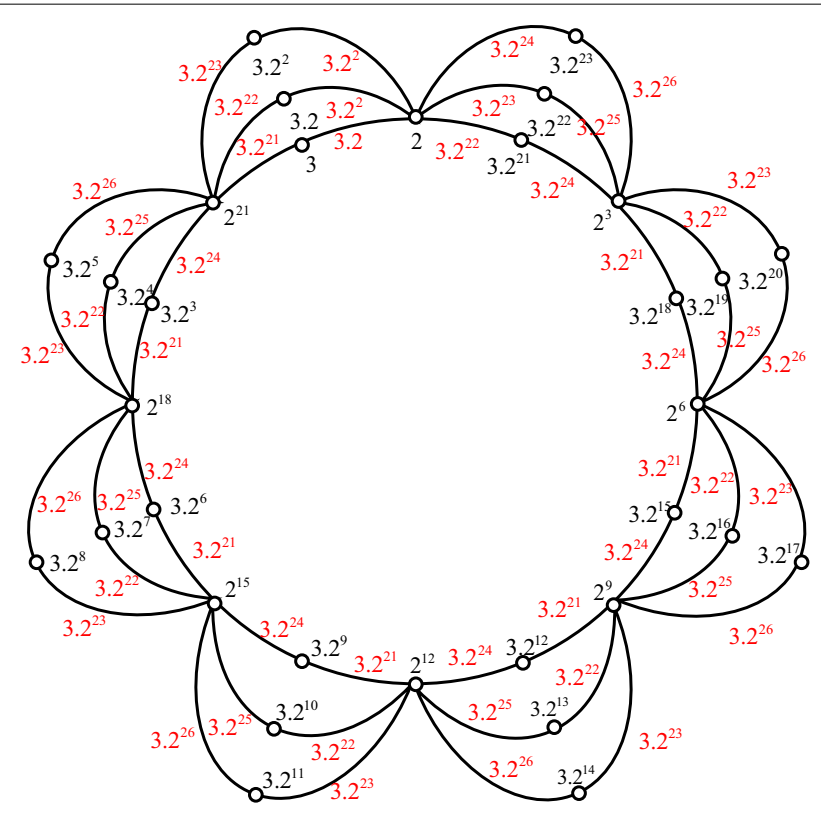

Fig. 2 9-Zumkeller labeling of $\operatorname{SSD}\left(C_{8}\right)$

that the graph $\operatorname{SSD}\left(P_{n} \odot K_{1}\right)$ has $2 t n+(2 n-t)$ vertices and $4 t n-2 t$ edges. Define labeling $f: V\left(\operatorname{SSD}\left(P_{n} \odot K_{1}\right)\right) \rightarrow N$ as follows:

For $1 \leq k \leq t$

$$
\begin{aligned}
f\left(u_{i}\right) & =2^{2 t i+(1-t)}, 1 \leq i \leq n \\
f\left(v_{i}\right) & =2^{2 t i+(1-2 t)}, 1 \leq i \leq n \\
f\left(u_{i(i+1)}^{k}\right) & =p 2^{2 t(n-i)-(1+t)+k}, 1 \leq i \leq n-1 \\
f\left(w_{i}^{k}\right) & =p 2^{2 t(n-i)+(k-1)}, 1 \leq i \leq n-1 .
\end{aligned}
$$

Where $p<10, p \neq 2$ is a prime number. So the labels of the edges of $\operatorname{SSD}\left(P_{n} \odot K_{1}\right)$ are given as follows:

For $1 \leq i \leq n-1,1 \leq k \leq t$

$$
\begin{aligned}
& f^{*}\left(u_{i} u_{i(i+1)}^{k}\right)=f^{*}\left(w_{i}^{k} v_{i}\right)=f^{*}\left(w_{n}^{k} v_{n}\right)=p 2^{2 t(n-1)+k}, \\
& f^{*}\left(u_{i(i+1)}^{k} u_{(i+1)}\right)=p 2^{2 t n+k} \\
& f^{*}\left(u_{i} w_{i}^{k}\right)=p 2^{t(2 n-1)+k} .
\end{aligned}
$$

From Eqs. (6) to (8), it is noticed that the edges of $\operatorname{SSD}\left(P_{n} \odot K_{1}\right)$ receive only $3 t$ distinct Zumkeller numbers $p 2^{2 t(n-1)+k}, p 2^{2 t n+k}$ and $p 2^{t(2 n-1)+k}$ for $1 \leq k \leq t$. Hence $\operatorname{SSD}\left(P_{n} \odot K_{1}\right)$ graph admits a $3 t$-Zumkeller labeling. 


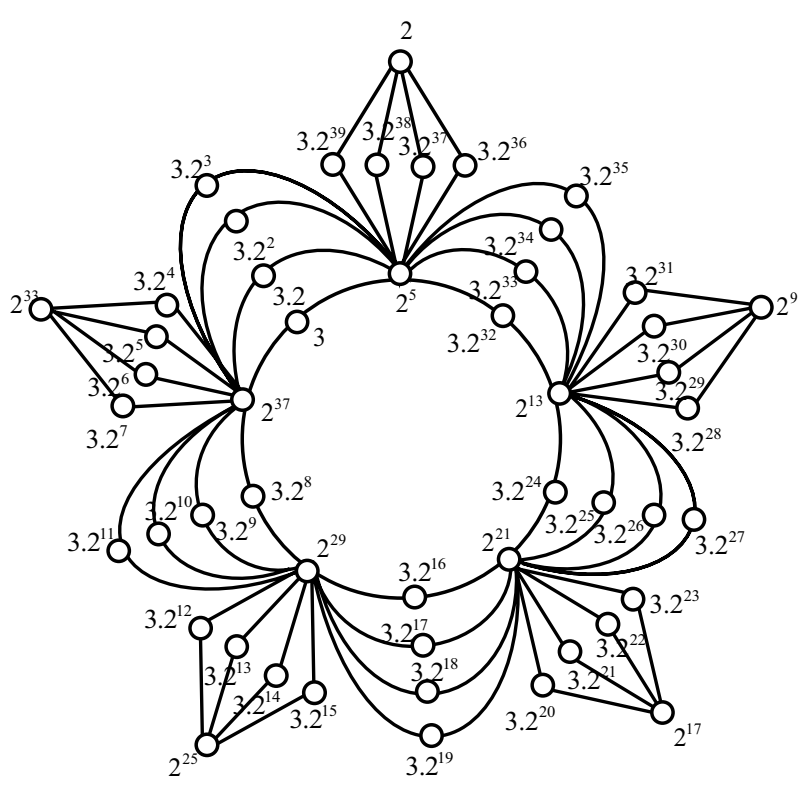

Fig. 3 15-Zumkeller labeling of $\operatorname{SSD}\left(P_{5} \odot K_{1}\right)$

Illustration 3.3 Figure 3 illustrates the super subdivision of $P_{5} \odot K_{1}$ and its 15-Zumkeller labeling, where $t=4$ and $p=3$.

Theorem 3.4 $\operatorname{SSD}\left(L_{n}\right)$ admits a $4 t$-Zumkeller labeling for all $n \geq 3, t \geq 1$.

Proof We get the ladder graph $L_{n}$ by attaching the vertices $v_{i}$ and $v_{i+1}$ for $1 \leq i \leq n-1$ of the comb graph $P_{n} \odot K_{1}$ in Theorem 3.3, with additional edges. Let $V=\left\{u_{i}, v_{i}: 1 \leq i \leq n\right\}$ be the vertex set and $E=\left\{e_{i}=u_{i} u_{i+1}, \hat{e}_{i}=u_{i} v_{i}, \bar{e}_{i}=v_{i} v_{i+1}: 1 \leq i \leq n-1\right\} \bigcup\left\{\hat{e}_{n}=u_{n} v_{n}\right\} \quad$ be $\quad$ the edge set of the ladder graph. By the definition of super subdivision of $L_{n}$, we can consider $u_{i(i+1)}^{k}, v_{i(i+1)}^{k}, w_{i}^{k}(1 \leq i \leq n-1,1 \leq k \leq t)$ and $w_{n}^{k}$ are the vertices of the $t$-vertices part of $k_{2, t}$. Then, it is clear that $\operatorname{SSD}\left(L_{n}\right)$ has $3 t n+2(n-t)$ vertices and $6 t n-4 t$ edges. Define labeling $f: V\left(S S D\left(L_{n}\right)\right) \rightarrow N$ as follows:

For $1 \leq i \leq n, 1 \leq k \leq t$

$$
\begin{aligned}
& f\left(u_{i}\right)= \begin{cases}2^{3 t i+(1-3 t)}, & i \text { is odd } \\
2^{3 t i+(1-2 t)}, & i \text { is even. }\end{cases} \\
& f\left(v_{i}\right)= \begin{cases}2^{3 t i+(1-2 t)}, & i \text { is odd } \\
2^{3 t i+(1-3 t)}, & i \text { is even. }\end{cases}
\end{aligned}
$$

$f\left(w_{i}^{k}\right)=p 2^{3 t(n-i)+k-1}$.

For $1 \leq i \leq n-1$. 


$$
\begin{aligned}
& f\left(u_{i(i+1)}^{k}\right)= \begin{cases}p 2^{3 t(n-i)-(2 t+1)+k}, & i \text { is odd } \\
p 2^{3 t(n-i)-(t+1)+k}, & i \text { is even. }\end{cases} \\
& f\left(v_{i(i+1)}^{k}\right)= \begin{cases}p 2^{3 t(n-i)-(t+1)+k}, & i \text { is odd } \\
p 2^{3 t(n-i)-(2 t+1)+k}, & i \text { is even. }\end{cases}
\end{aligned}
$$

Where $p$ is a prime number greater than 2 but less than 10. Therefore, the labels of the edges of $\operatorname{SSD}\left(L_{n}\right)$ are given as follows:

For $1 \leq i \leq n-1$

$$
\begin{aligned}
& f^{*}\left(u_{i} u_{i(i+1)}^{k}\right)= \begin{cases}p 2^{3 t n-5 t+k}, & i \text { is odd } \\
p 2^{3 t n-3 t+k}, & i \text { is even. }\end{cases} \\
& f^{*}\left(v_{i} v_{i(i+1)}^{k}\right)= \begin{cases}p 2^{3 t n-3 t+k}, & i \text { is odd } \\
p 2^{3 t n-5 t+k}, & i \text { is even. }\end{cases} \\
& f^{*}\left(u_{i(i+1)}^{k} u_{(i+1)}\right) *=f^{*}\left(v_{i(i+1)}^{k} v_{(i+1)}\right)=p 2^{3 t n-t+k} .
\end{aligned}
$$

For $1 \leq i \leq n$.

$$
\begin{aligned}
f^{*}\left(u_{i} w_{i}^{k}\right) & = \begin{cases}p 2^{3 t n-3 t+k}, & i \text { is odd } \\
p 2^{3 t n-2 t+k}, & i \text { is even. }\end{cases} \\
f^{*}\left(v_{i} w_{i}^{k}\right) & = \begin{cases}p 2^{3 t n-2 t+k}, & i \text { is odd } \\
p 2^{3 t n-3 t+k}, & i \text { is even. }\end{cases}
\end{aligned}
$$

From Eqs. (9) to (13), it is observed that the edges of $\operatorname{SSD}\left(L_{n}\right)$ receive only $4 t$ distinct Zumkeller numbers $p 2^{3 t n-3 t+k}, p 2^{3 t n-5 t+k}, p 2^{3 t n-2 t+k}$ and $p 2^{3 t n-t+k}$ for $1 \leq k \leq t$. Hence $S S D\left(L_{n}\right)$ graph admits a $4 t-$ Zumkeller labeling.

Illustration 3.4 Figure 4 illustrates the super subdivision of $\operatorname{SSD}\left(L_{5}\right)$ and its 12-Zumkeller labeling where, $t=3$ and $p=3$.

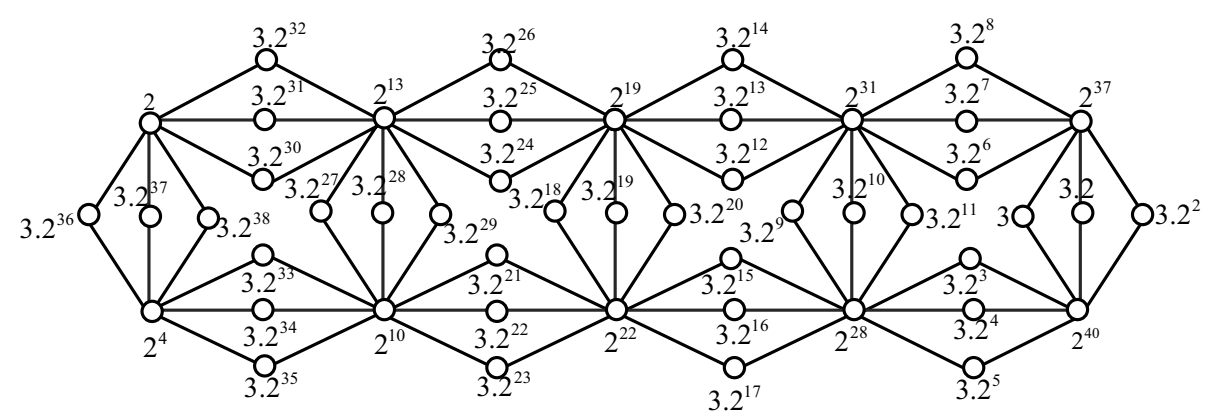

Fig. 4 12-Zumkeller labeling of $S S D\left(L_{5}\right)$ 
Theorem 3.5 $\operatorname{SSD}\left(C_{n} \odot K_{1}\right)$ admits a 4 t-Zumkeller labeling for all $n \geq 2$.

Proof Let $V=\left\{u_{i}, v_{i}: 1 \leq i \leq n\right\}$ be the vertex set and $E=\left\{e_{i}=u_{i} u_{i+1}, \hat{e}_{i}=u_{i} v_{i}: 1 \leq i \leq n-1\right\} \bigcup\left\{e_{n}=u_{n} u_{1}, \hat{e}_{n}=u_{n} v_{n}\right\}$ be the edge set of the crown graph. Suppose that $u_{i(i+1)}^{k}, w_{i}^{k}(1 \leq i \leq n-1,1 \leq k \leq t), u_{n 1}^{k}$ and $w_{n}^{k}$ are the vertices of the $t$-vertices part of $K_{2, t}$ which are used for super subdivision of $C_{n} \odot K_{1}$. Then, it obvious that the graph $\operatorname{SSD}\left(C_{n} \odot K_{1}\right)$ has $2 t n+2 n$ vertices and $4 t n$ edges. The labels of vertices $u_{i}, v_{i}$ for $1 \leq i \leq n$ are given as in Theorem 3.4. For the vertices $u_{i(i+1)}^{k}, w_{i}^{k}$ and $u_{n 1}^{k}$, we define the vertex function $f$ as follows:

For $1 \leq k \leq t$

$$
\begin{aligned}
f\left(u_{i(i+1)}^{k}\right) & =p 2^{2 t(n-i)+k-1}, \quad 1 \leq i \leq n-1 \\
f\left(w_{i}^{k}\right) & =p 2^{2 t(n-i)+(t-1)+k}, \quad 1 \leq i \leq n \\
f\left(u_{n 1}^{k}\right) & =p 2^{k-1} .
\end{aligned}
$$

Where $p<10, p \neq 2$ is a prime number. Now the labels of the edges of $\operatorname{SSD}\left(C_{n} \odot K_{1}\right)$ are given as follows:

For $1 \leq i \leq n-1$

$$
\begin{aligned}
& f^{*}\left(u_{i} u_{i(i+1)}^{k}\right)=f^{*}\left(u_{n} u_{n 1}^{k}\right)=p 2^{2 t n-t+k} \\
& f^{*}\left(w_{i}^{k} v_{i}\right)=f^{*}\left(w_{n}^{k} v_{n}\right)=p 2^{2 t n-t+k} \\
& f^{*}\left(u_{i(i+1)}^{k} u_{(i+1)}\right)=p 2^{2 t n+t+k} \\
& f^{*}\left(u_{n 1}^{k} u_{1}\right)=p 2^{t+k} \\
& f^{*}\left(u_{i} w_{i}^{k}\right)=p 2^{2 t n+k} .
\end{aligned}
$$

From Eqs. (14) to (18), it is noticed that the edges of $\operatorname{SSD}\left(C_{n} \odot K_{1}\right)$ receive only $4 t$ distinct Zumkeller numbers $p 2^{2 t n-t+k}, p 2^{2 t n+k-1}, p 2^{t+k}$ and $p 2^{2 t n+k}$ for $1 \leq k \leq t$. Hence $\operatorname{SSD}\left(C_{n} \odot K_{1}\right)$ graph admits a $4 t$-Zumkeller labeling.

Illustration 3.5 Figure 5 illustrates the super subdivision of $\operatorname{SSD}\left(C_{5} \odot K_{1}\right)$ and its 16-Zumkeller labeling where, $t=4$ and $p=3$.

Theorem 3.6 For $n \geq 2, \operatorname{SSD}\left(C L_{n}\right)$ admits a 6 -Zumkeller labeling if $n$ is odd and 5 t-Zumkeller labeling if $n$ is even. 


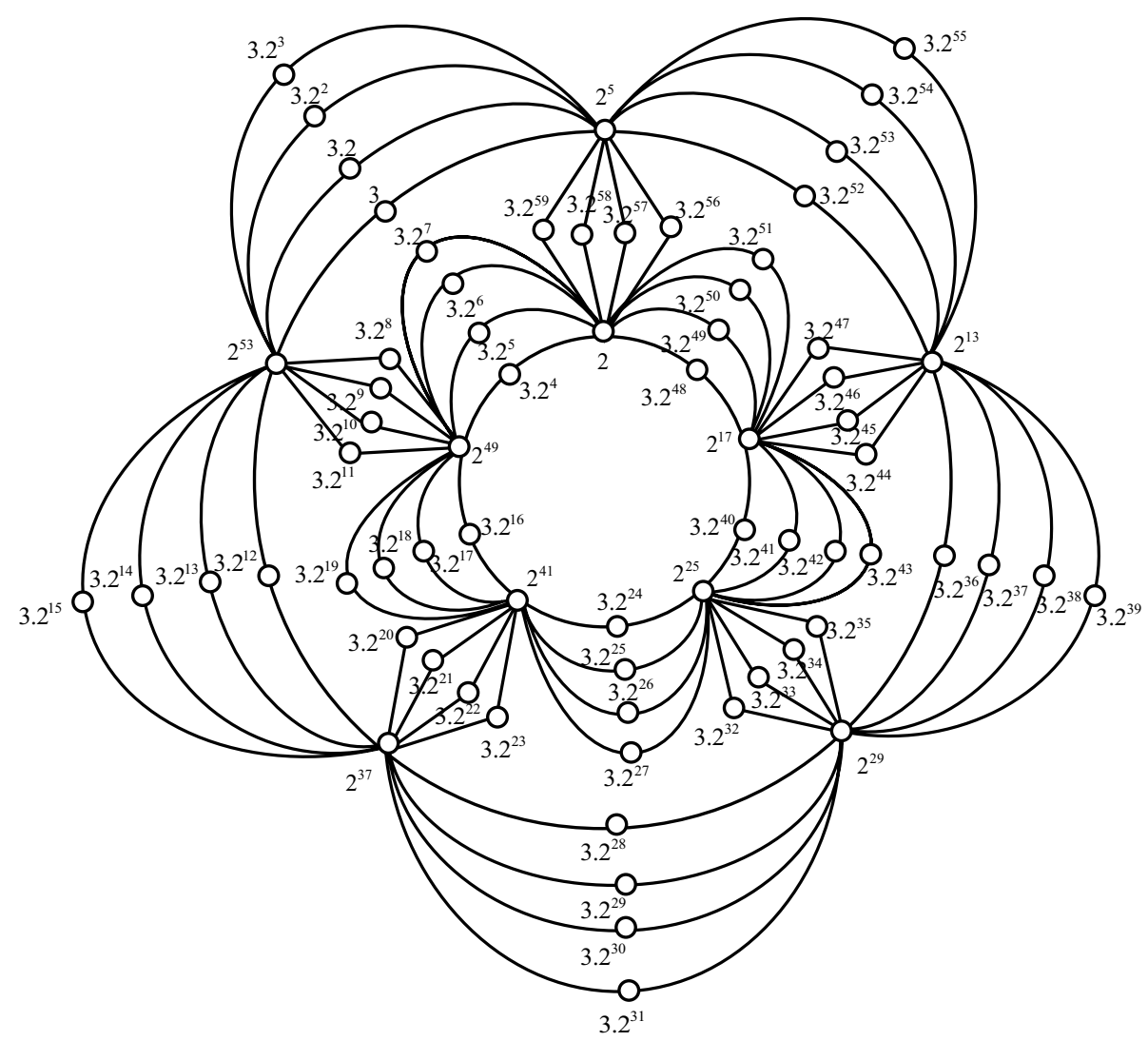

Fig. 5 16-Zumkeller labeling of $\operatorname{SSD}\left(C_{8} \odot K_{1}\right)$

Proof By joining the vertices $u_{n}, u_{1}$ and $v_{n}, v_{1}$ of the ladder graph $L_{n}$ in Theorem 3.5, with additional edges we get the circular ladder graph $C L_{n}$. Let $V=\left\{u_{i}, v_{i}: 1 \leq i \leq n\right\}$ be the vertex set and $E=\left\{e_{i}=u_{i} u_{i+1}, \bar{e}_{i}=v_{i} v_{i+1}, \hat{e}_{i}=u_{i} v_{i}: 1 \leq i \leq n-1\right\} \bigcup\left\{e_{n}=u_{n} u_{1}, \overline{e_{n}}=\right.$ $\left.u_{n} u_{1}, \hat{e}_{n}=u_{n} v_{n}\right\}$ - be the edge set of the circular graph. Let $S D D\left(C L_{n}\right)$ be the graph obtained by super subdivision of $C L_{n}$ by a complete bipartite graph $K_{2, t}$. Thus, $S D D\left(C L_{n}\right)$ has $3 t n+2 n$ vertices and $6 t n$ edges. The labels of vertices $u_{i}, v_{i}$ for $1 \leq i \leq n$ are given as in Theorem 3.5. For the vertices $u_{i(i+1)}^{k}, v_{i(i+1)}^{k}$ and $w_{i}^{k}$ we define the vertex function $f$ as follows:

Case (I): If $n$ is odd.

For $1 \leq i \leq n-1,1 \leq k \leq t$. 


$$
\begin{aligned}
f\left(u_{i(i+1)}^{k}\right) & = \begin{cases}p 2^{3 t(n-i)+k-1}, & i \text { is odd } \\
p 2^{3 t(n-i)+(t-1)+k}, & i \text { is even. }\end{cases} \\
f\left(v_{i(i+1)}^{k}\right) & = \begin{cases}p 2^{3 t(n-i)+(t-1)+k}, & i \text { is odd } \\
p 2^{3 t(n-i)+k-1}, & i \text { is even. }\end{cases} \\
f\left(w_{i}^{k}\right) & =p 2^{3 t(n-i)+(2 t-1)+k} \\
f\left(u_{n 1}^{k}\right) & =p 2^{k+t-1} \\
f\left(v_{n 1}^{k}\right) & =p 2^{k} .
\end{aligned}
$$

where $p$ is a prime number greater than 2 but less than 10 . Then the labels of the edges of $\operatorname{SSD}\left(C L_{n}\right)$ are given as follows:

$$
\begin{aligned}
& f^{*}\left(u_{i} u_{i(i+1)}^{k}\right)= \begin{cases}p 2^{3 t n-3 t+k}, & i \text { is odd } \\
p 2^{3 t n-t+k}, & i \text { is even. }\end{cases} \\
& f^{*}\left(v_{i} v_{i(i+1)}^{k}\right)= \begin{cases}p 2^{3 t n-t+k}, & i \text { is odd } \\
p 2^{3 t n-3 t+k}, & i \text { is even. }\end{cases} \\
& f^{*}\left(u_{i} w_{i}^{k}\right)= \begin{cases}p 2^{3 t n-t+k}, & i \text { is odd } \\
p 2^{3 t n+k}, & i \text { is even. }\end{cases} \\
& f^{*}\left(v_{i} w_{i}^{k}\right)= \begin{cases}p 2^{3 t n+k}, & i \text { is odd } \\
p 2^{3 t n-t+k}, & i \text { is even. }\end{cases} \\
& f^{*}\left(v_{n} v_{n 1}^{k}\right)=f^{*}\left(u_{n} u_{n 1}^{k}\right)=p 2^{3 t n-2 t+k} \\
& f^{*}\left(u_{i(i+1)}^{k} u_{(i+1)}\right)=f^{*}\left(v_{i(i+1)}^{k} v_{(i+1)}\right)=p 2^{3 t n+t+k} \\
& f^{*}\left(u_{n 1}^{k} u_{1}\right)=f^{*}\left(v_{n 1}^{k} v_{1}\right)=p 2^{k+t} .
\end{aligned}
$$

From Eqs. (19) to (25), it is noticed that the edges of $\operatorname{SSD}\left(C L_{n}\right)$ receive only $6 t$ distinct Zumkeller numbers $p 2^{3 t n-3 t+k}, p 2^{3 t n-t+k}, p 2^{3 t n+k}, p 2^{3 t n+t+k}$,

$p 2^{k+1}$ and $p 2^{3 t n-2 t+k}$ for $1 \leq k \leq t$. Hence $\operatorname{SSD}\left(C L_{n}\right)$ graph admits a $6 t$-Zumkeller labeling when $n$ is odd.

Case (II): If $n$ is even. It is easy verified that the edge labels of $u_{n} u_{n 1}^{k}, v_{n} v_{n 1}^{k}$ equal to the edge labels of $v_{i} w_{i}^{k}, v_{i} v_{(i(i+1)}^{k}$, respectively, for $i$ is even. Then, the edges of $\operatorname{SSD}\left(C L_{n}\right)$ receive only $5 t$ distinct Zumkeller numbers $p 2^{3 t n-3 t+k}, p 2^{3 t n-t+k}, p 2^{3 t n+k}, p 2^{3 t n+t+k}$

and $p 2^{k+1}$ for $1 \leq k \leq t$. Hence $S S D\left(C L_{n}\right)$ graph admits a $5 t$-Zumkeller labeling when $n$ is even.

Illustration 3.6 Figure 6 illustrates the super subdivision of $\operatorname{SSD}\left(C L_{5}\right)$ and its 24-Zumkeller labeling where, $t=4$ and $p=3$. 


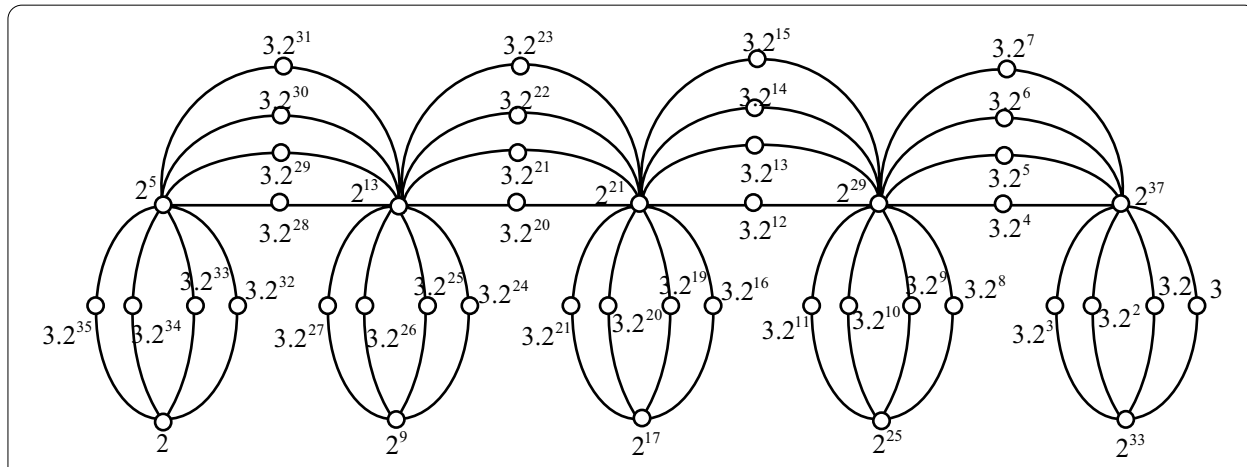

Fig. 6 24-Zumkeller labeling of $S S D\left(C L_{5}\right)$



Fig. 7 15-Zumkeller labeling of $\operatorname{SSD}\left(\mathrm{CL}_{4}\right)$

Illustration 3.7 Figure 7 illustrates the super subdivision of $\operatorname{SSD}\left(C L_{4}\right)$ and its 15-Zumkeller labeling where, $t=3$ and $p=3$.

Theorem 3.7 For $\operatorname{SSD}\left(P_{m} \times P_{n}\right)$ admits a $2 m$ t-Zumkeller labeling for all $m, n \geq 3$.

Proof Let $V=\left\{u_{i j}: 1 \leq i \leq m, 1 \leq j \leq n\right\}$ be the vertex set and $E=\left\{e_{i j}=u_{i j} u_{i(j+1)}, \hat{e}_{i j}=u_{i j} u_{(i+1) j}: 1 \leq i \leq m-1,1 \leq j \leq n-1\right\}$ be the edge set of the planar grid $P_{m} \times P_{n}$. Let $S S D\left(P_{m} \times P_{n}\right)$ be the graph obtained by super subdivision of $P_{m} \times P_{n}$ in which the edges $e_{i j}, \hat{e}_{i j}$ of $P_{m} \times P_{n}$ are replaced by a complete bipartite graph $k_{2, t}$. Let $u_{i, j(j+1)}^{k}, u_{i(i+1), j}^{k}$ be the vertices of $t$-vertices part where $1 \leq i \leq m-1,1 \leq j \leq n-1$ and $1 \leq k \leq t$. Thus, graph $G=\operatorname{SSD}\left(P_{m} \times P_{n}\right)$ has $m n(1+2 t)-(m+n) t$ vertices and $4 m n t-2 t(m+n)$ edges. Define labeling $f: V\left(S S D\left(P_{m} \times P_{n}\right)\right) \rightarrow N$ as follows: For $1 \leq k \leq t$. 


$$
\begin{array}{rlrl}
f\left(u_{i j}\right) & =2^{1+n t(i-1)+t(j-1)} \quad 1 \leq i \leq m, 1 \leq j \leq n \\
f\left(u_{i, j(j+1)}^{k}\right) & =p 2^{(2 m n-m+n-1) t-(2 n-1) t i-t j+(k-1)}, & & 1 \leq i \leq m, 1 \leq j \leq n-1 \\
f\left(u_{i(i+1), j}^{k}\right) & =p 2^{(2 m n-m) t-(2 n-1) t i-t j+(k-1)}, & & 1 \leq i \leq m-1,1 \leq j \leq n .
\end{array}
$$

where $p<10, p \neq 2$ is a prime number. Thus the labels of the edges of $\operatorname{SSD}\left(P_{m} \times P_{n}\right)$ are given as follows:

$$
\begin{array}{ll}
f^{*}\left(u_{i j} u_{i, j(j+1)}^{k}\right)=p 2^{(2 m n-m-2) t-(n-1) t i+k}, & 1 \leq i \leq m \\
f^{*}\left(u_{i, j(j+1)}^{k} u_{i(j+1)}\right)=p 2^{(2 m n-m-1) t-(n-1) t i+k} & 1 \leq i \leq m \\
f^{*}\left(u_{i j} u_{i(i+1), j}^{k}\right)=p 2^{(2 m n-m-n-1) t-(n-1) t i+k}, & 1 \leq i \leq m-1 \\
f^{*}\left(u_{i(i+1), j}^{k} u_{(i+1) j}\right)=p 2^{(2 m n-m-1) t-(n-1) t i+k}, & 1 \leq i \leq m-1 .
\end{array}
$$

From Eqs. (26) to (29), it is observed that the edges of $\operatorname{SSD}\left(P_{m} \times P_{n}\right)$ receive Zumkeller numbers and the numbers $p 2^{(2 m n-m-n-1) t-(n-1) t i+k}, 1 \leq i \leq m-1$ are equal to the numbers $p 2^{(2 m n-m-2) t-(n-1) t i+k}, 2 \leq i \leq m$. From Table 1 , we found that the number of Zumkeller numbers used to label the edges is $2 m t$.

Illustration 3.8 Figure 8 illustrates the super subdivision of $\operatorname{SSD}\left(P_{3} \times P_{5}\right)$ and its 24-Zumkeller labeling where, $t=4$ and $p=3$.

Theorem 3.8 $\operatorname{SSD}\left(C_{m} \times P_{n}\right)$ admits a $(2 m+1)$ t-Zumkeller labeling for all $m, n \geq 3$.

Proof By joining the vertices $u_{i 1}$ and $u_{i n}, \quad 1 \leq i \leq m$ of the planar grid $\left(P_{m} \times P_{n}\right)$ in Theorem 3.7, we obtain the prism graph $C_{m} \times P_{n} . \quad$ Let $\quad V\left(C_{m} \times P_{n}\right)=\left\{u_{i j}, 1 \leq i \leq m, 1 \leq j \leq n\right\} \quad$ and

Table 1 Zumkeller numbers used to label the edges of $\operatorname{SSD}\left(P_{m} \times P_{n}\right)$

\begin{tabular}{lll}
\hline Edges & Labels & Num. of labels \\
\hline$u_{1 j} u_{1, j(j+1)}^{k}$ & $p 2^{(2 m n-m-n-1) t+k}$ & $(n-1) t$ \\
$u_{i j} u_{i, j(j+1)^{\prime}}^{k}$ & $p 2^{(2 m n-m-1) t-(n-1) t i+k}$ & $(m-1)(n-1) t$ \\
$u_{(i-1) j} u_{(i-1) i j^{\prime}}$ & & \\
$2 \leq i \leq m$ & $p 2^{(2 m n-m-1) t-(n-1) t i+k}$ & $(m-1)(n-1) t$ \\
$u_{i j(j+1)}^{k} u_{i(j+1),}$ & & \\
$u_{i(i+1) j}^{k^{\prime}} u_{(i+1) j}$ & & $(n-1) t$ \\
$1 \leq i \leq m-1$ & $p 2^{(m n-1) t+k}$ & \\
$u_{m, j(j+1)}^{k} u_{m(j+1)}$ & & \\
\hline
\end{tabular}




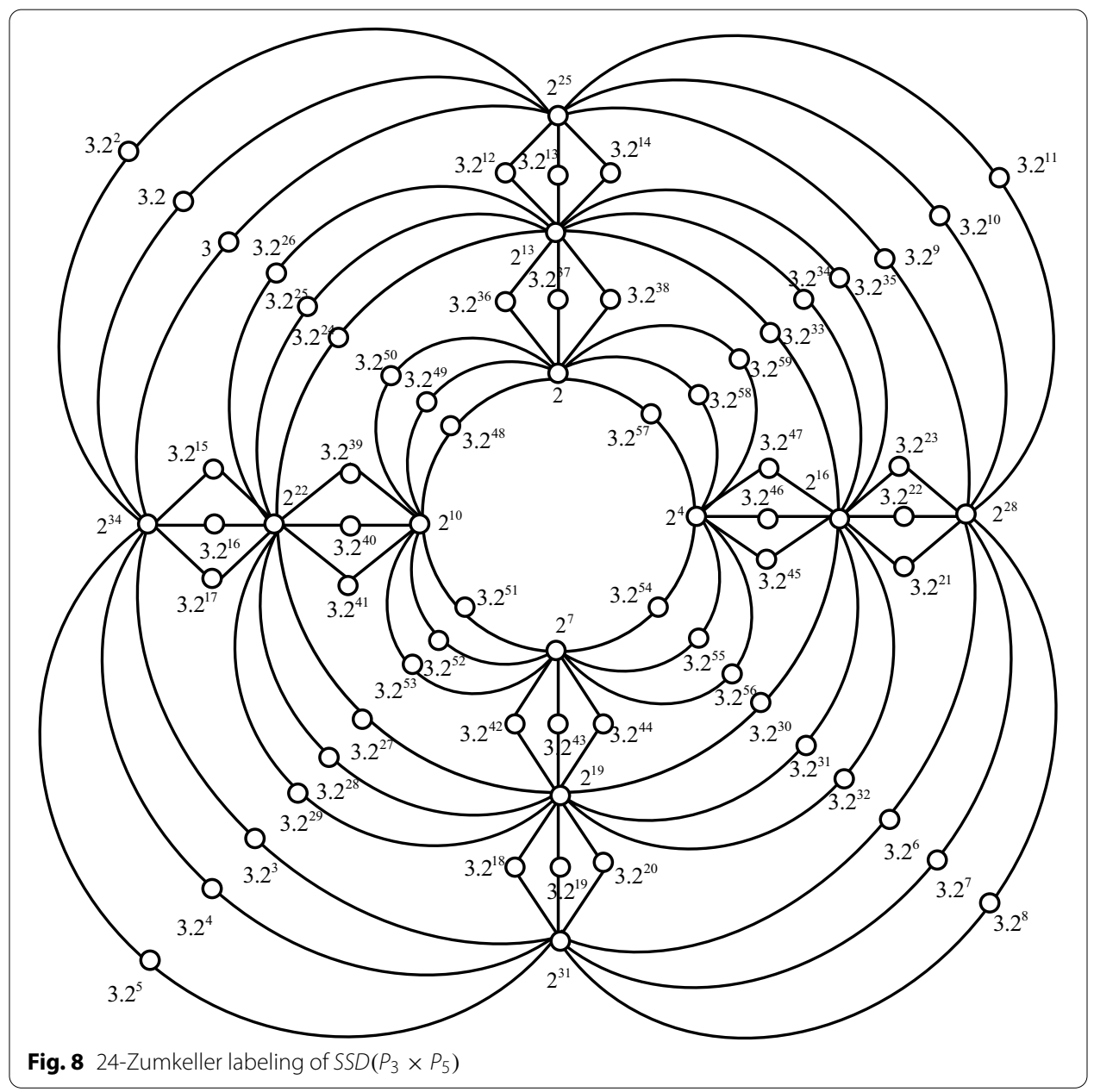

$E=\left\{e_{i j}=u_{i j} u_{i(j+1)}, \hat{e}_{i j}=u_{i j} u_{(i+1) j}: 1 \leq i \leq m-1,1 \leq j \leq n-1\right\} \bigcup\left\{e_{i n}=\right.$ $\left.u_{i 1} u_{i n}, 1 \leq i \leq m\right\}$

the definition of super subdivision of $C_{m} \times P_{n}$, we can consider $u_{i, j(j+1)}^{k}, u_{i(i+1), j}^{k}, u_{i(i+1), n}^{k}$ and $u_{i, n 1}^{k}$ are the vertices of $t$-vertices part where $1 \leq i \leq m-1,1 \leq j \leq n-1$ and $1 \leq k \leq t$. Hence, it is clear that $\operatorname{SSD}\left(C_{m} \times P_{n}\right)$ has $m n(1+2 t)-(m+n) t$ vertices and $4 m n t-2 n t$ edges. The labels of vertices $u_{i j}$ for $1 \leq i \leq m, 1 \leq j \leq n$ are given as in Theorem 3.7. For the vertices $u_{i, j(j+1)}^{k}, u_{i(i+1), j}^{k}, u_{i(i+1), n}^{k}$ and $u_{i, n 1}^{k}$ we define the vertex function $f$ as follows:

For $1 \leq k \leq t$

$$
\begin{aligned}
f\left(u_{i, j(j+1)}^{k}\right) & =p 2^{(2 m n+n) t-2 n t i-t j+k-1}, \quad 1 \leq i \leq m, 1 \leq j \leq n-1 \\
f\left(u_{i, n 1}^{k}\right) & =p 2^{2 m n t-2 n t i+k-1}, \quad 1 \leq i \leq m \\
f\left(u_{i(i+1), 1}^{k}\right) & =p 2^{(2 m n-n) t-2 n t i+k-1}, \quad 1 \leq i \leq m-1 \\
f\left(u_{i(i+1), j}^{k}\right) & =p 2^{(2 m n+1) t-2 n t i-t j+k-1}, \quad 1 \leq i \leq m-1,2 \leq j \leq n .
\end{aligned}
$$

Where $p<10, p \neq 2$ is a prime number. Then the labels of the edges of $\operatorname{SSD}\left(C_{m} \times P_{n}\right)$ are given as follows: 
For $1 \leq i \leq m, 1 \leq j \leq n-1$.

$$
\begin{aligned}
& f^{*}\left(u_{i j} u_{i, j(j+1)}^{k}\right)=f^{*}\left(u_{i n} u_{i, n 1}^{k}\right)=p 2^{(2 m n-1) t-n t i+k} \\
& f^{*}\left(u_{i, j(j+1)}^{k} u_{i(j+1)}\right)=p 2^{2 m n t-n t i+k} \\
& f^{*}\left(u_{i, n 1}^{k} u_{i 1}\right)=p 2^{n(2 m-1) t-n t i+k} .
\end{aligned}
$$

For $1 \leq i \leq m-1,2 \leq j \leq n$.

$$
\begin{aligned}
& f^{*}\left(u_{i 1} u_{i(i+1), 1}^{k}\right)=p 2^{2 m n t-n t(i+2)+k} \\
& f^{*}\left(u_{i j} u_{i(i+1), j}^{k}\right)=f^{*}\left(u_{i(i+1), 1}^{k} u_{(i+1) 1}\right)=p 2^{n(2 m-1) t-n t i+k} \\
& f^{*}\left(u_{i(i+1), j}^{k} u_{(i+1) j}\right)=p 2^{2 m n t-n t i+k} .
\end{aligned}
$$

\begin{tabular}{|c|c|c|c|}
\hline Edges & Num. of edges & Labels & Num. of labels \\
\hline $\begin{array}{l}u_{i j} u_{i, j(j+1)^{\prime}} \\
u_{i n} u_{i, n 1}^{k} \\
1 \leq i \leq m, \\
1 \leq j \leq n-1\end{array}$ & $m n t$ & $p 2^{(2 m n-1) t-n t i+k}$ & $m t$ \\
\hline $\begin{array}{l}u_{i, j(j+1)}^{k} u_{i(j+1)} \\
1 \leq i \leq m, \\
1 \leq j \leq n-1 \\
u_{(i-1) j} u_{(i-1) i j}^{k} \\
2 \leq i \leq m_{1} \\
2 \leq j \leq n-1 \\
u_{(i-1), n 1}^{k} u_{(i-1) 1} \\
2 \leq i \leq m \\
u_{i(i+1) j}^{k} u_{(i+1) j} \\
1 \leq i \leq m, \\
2 \leq j \leq n-1 \\
u_{(i-2) 1} u_{(i-2)(i-1), 1}^{k} \\
3 \leq i \leq m, \\
1 \leq j \leq n-1 \\
u_{(i-1) i, 1}^{k} u_{i j} \\
2 \leq i \leq m\end{array}$ & $\begin{array}{l}(n-1) m t \\
(n-1)(m-1) t \\
(m-1) t \\
(m-1) t \\
(m-2) t \\
(m-1) t\end{array}$ & $p 2^{2 m n t-n t i+k}$ & $m t$ \\
\hline $\begin{array}{l}u_{m, n 1}^{k} u_{m 1} \\
u_{(m-1) 1} u_{(m-1) m, 1}^{k}\end{array}$ & $2 t$ & $p 2^{m n t-n t+k}$ & t \\
\hline
\end{tabular}

Table 2 Zumkeller numbers used to label the edges of $\operatorname{SSD}\left(C_{m} \times P_{n}\right)$ 


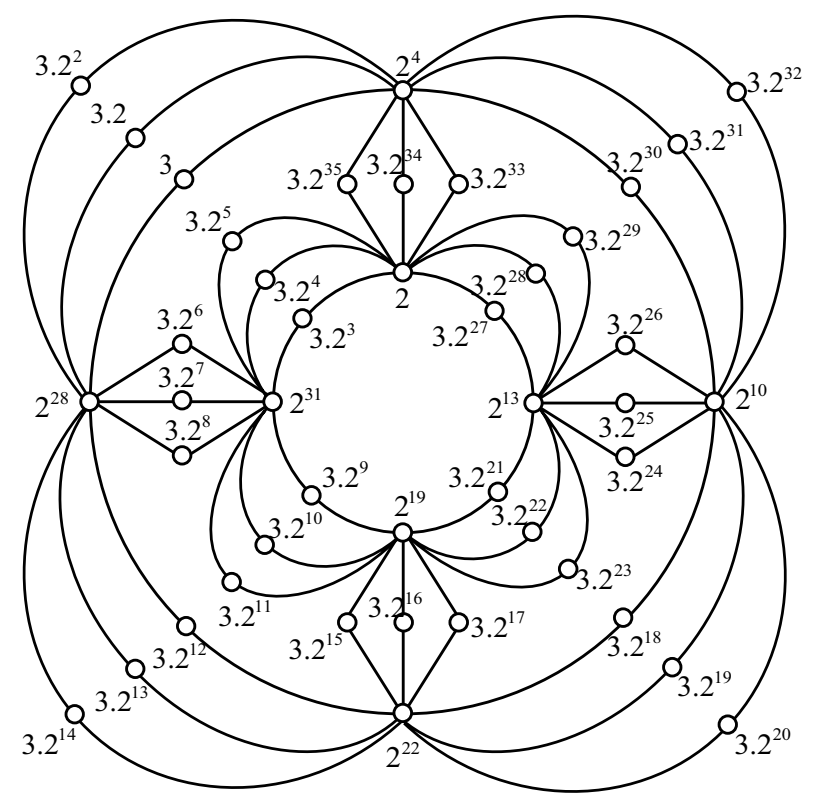

Fig. 9 21-Zumkeller labeling of $\operatorname{SSD}\left(C_{3} \times P_{4}\right)$

From Eqs. (30) to (35), it is observed that the edges of $S S D\left(C_{m} \times P_{n}\right)$ receive Zumkeller numbers. Now the total number of edges is $4 m n t-2 n t$ and from Table 2 we found that the number of Zumkeller numbers used to label the edges is $(2 m+1) t$.

Illustration 3.9 Figure 9 illustrates the super subdivision of $\operatorname{SSD}\left(C_{3} \times P_{4}\right)$ and its 21-Zumkeller labeling where, $t=3$ and $p=3$.

\section{Conclusions}

From the previous sections, we studied the $k$-Zumkeller labeling for some graphs. For any natural number $n \geq 2$, we show that the super subdivision of the path $P_{n}$ is $2 t$-Zumkeller graph, while the super subdivision of the cycle $C_{n}$ and the comb $P_{n} \odot K_{1}, n \geq 3$ are $3 t$-Zumkeler graphs. Also, we prove that the super subdivision of the ladder $L_{n}, n \geq 3$ and the crown $C_{n} \odot K_{1}, n \geq 2$ admit $4 t$-Zumkeller labeling. Moreover, we show that the super subdivision of the circular ladder $C L_{n}, n \geq 2$ has a $6 t$-Zumkeller labeling when $n$ is odd and a $5 t$-Zumkeller labeling when $n$ is even. Finally, for $m, n \geq 3$ we prove that the planer grid $P_{m} \times P_{n}$ is $2 m t$-Zumkeller graph, however the prism $C_{m} \times P_{n}$ is $(2 m+1) t$ -Zumkeller graph, where $t$ is the number of vertices of $t$-vertices part.

Abbreviations

SSD(G): Super subdivision of graph G.

Acknowledgements

Not applicable. 


\section{Authors' contributions}

MB carried out all theorems, proofs and drafted the manuscript by himself without any participation. All authors read and approved the final manuscript.

Funding

No funding was received.

Availability of data and materials

Data sharing not applicable to this article as no data sets were generated or analyzed during the current study. Data sharing not applicable to this article as no data sets were generated or analyzed during the current study.

\section{Declarations}

\section{Competing interests}

The author declare that no competing interests.

Received: 19 December 2019 Accepted: 26 April 2021

Published online: 19 May 2021

\section{References}

1. Rosa, A.: On certain valuations of the vertices of a graph. Theory Graphs 1967, 349-355 (1966)

2. Gallian, J.A.: A dynamic survey of graph labeling. Electron. J. Combinat., Twentieth edition, December 22, \#DS6 (2017)

3. Ahuja, R.K., Magnanti, T.L., Orlin, J.B.: Network Flows: Theory, Algorithms, and Applications. Prentice Hall (1993)

4. Harary, F.: Graph Theory. Addison-wesley, Reading Mass (1972)

5. Peng, Y., Bhaskara Rao, K.P.S.: On Zumkeller numbers. J. Number Theory 133(4), 1135-1155 (2013)

6. Balamurugan, B.J., Thirusangu, K., Thomas, D.G.: Strongly multiplicative Zumkeller labeling of graphs. In: International Conference on Information and Mathematical Sciences, pp. 349-354. Elsevier (2013)

7. Balamurugan, B.J., Thirusangu, K., Thomas, D.G., Murali, B.J.: k-Zumkeller labeling of graphs. Int. J. Eng. Technol. 7(4.10), 460-463 (2018)

8. Balamurugan, B.J., Thirusangu, K., Thomas, D.G.: k-Zumkeller labeling for twig graphs. Electron. Notes Discrete Math. 48, 119-126 (2015)

9. Sethuraman, G., Selvaraju, P.: Gracefulness of arbitrary of super subdivision of graphs. Indian J. Pure Appl. Math 32(7), 1059-1064 (2001). Natl. Acad. Sci. Lett. 26, 210-213 (2003)

10. Ascioglu, M., Cangul, I.: Narumi-Katayama index of the subdivision graphs. J. Taibah Univ. Sci. 12(4), $401-408$ (2018)

\section{Publisher's Note}

Springer Nature remains neutral with regard to jurisdictional claims in published maps and institutional affiliations.

\section{Submit your manuscript to a SpringerOpen ${ }^{\odot}$ journal and benefit from:}

Convenient online submission

Rigorous peer review

Open access: articles freely available online

- High visibility within the field

Retaining the copyright to your article

Submit your next manuscript at $\gg$ springeropen.com 